\title{
Seapolynol and Dieckol Improve Insulin Sensitivity through the Regulation of the PI3K Pathway in C57BL/KsJ-db/db Mice
}

\author{
Hui-Jeon Jeon ${ }^{1, \dagger}$, Kye-Yoon Yoon ${ }^{1, \dagger}$, Eun-Jeong Koh ${ }^{1}$, Jia Choi ${ }^{1}$, Kui-Jin Kim ${ }^{1}$, Hyeon-Son Choi ${ }^{2}$, Boo-Yong Lee ${ }^{1, *}$ \\ ${ }^{1}$ Department of Food Science and Biotechnology, CHA University, Seongnam, South Korea \\ ${ }^{2}$ Department of Food Science and Technology, Seoul Women’s University, Hwarang, Nowon, Seoul, 139-774, South Korea \\ ${ }^{\dagger}$ These authors contributed equally to this work. \\ *Corresponding author: bylee@cha.ac.kr
}

\begin{abstract}
Ecklonia cava, a brown alga native to the seas of Korea and Japan, contains biologically active polyphenols. Seapolynol (SN) is a polyphenol mixture derived from Ecklonia cava, and dieckol (DK) is a major compound of SN. Both Ecklonia cava-derived polyphenols exert an anti-diabetic effect in C57BL/KsJ-db/db mice. SN and DK supplementation significantly reduced bodyweight, water intake, fasting blood glucose, and plasma insulin levels compared with $\mathrm{db} / \mathrm{db}$ mice. In addition, C-peptide, a biomarker of insulin secretion, was significantly decreased by SN and DK treatment, whereas plasma adiponectin levels, which are associated with insulin sensitivity, was increased by two-fold compared with the $\mathrm{db} / \mathrm{db}$ group. Regarding plasma lipid profiles, triacylglycerol, total cholesterol, and low-density lipoprotein (LDL) cholesterol, were effectively reduced in the SN- and DK-treated groups. Moreover, the administration of SN and DK activated the insulin signaling pathway, as indicated by the levels of receptor substrate-1 (IRS-1), phosphoinositide 3-kinase (PI3K), serine-threonine specific protein kinase (Akt), and glucose transporter type 4 (GLUT4) in diabetic mice. This result showed that SN and DK regulate blood glucose levels by improving insulin sensitivity in the diabetic condition. Our data suggest that SN and DK may be anti-diabetic agents.
\end{abstract}

Keywords: Seapolynol, Dieckol, Type 2 diabetes, C57BL/KsJ-db/db mice, IRS-1

Cite This Article: Hui-Jeon Jeon, Kye-Yoon Yoon, Eun-Jeong Koh, Jia Choi, Kui-Jin Kim, Hyeon-Son Choi, and Boo-Yong Lee, "Seapolynol and Dieckol Improve Insulin Sensitivity through the Regulation of the PI3K Pathway in C57BL/KsJ-db/db Mice.” Journal of Food and Nutrition Research, vol. 3, no. 10 (2015): 648-652. doi: 10.12691/jfnr-3-10-5.

\section{Introduction}

Ecklonia cava is a brown alga native to the southern coasts of Korea and Japan. It contains an abundance of polyphenolic compounds, particularly phlorotannins, which have pharmaceutical potential [1,2]. The phlorotannins include compounds such as phloroglucinol, eckol, bieckol, fucodiphloroethol G, and dieckol. Seapolynol (SN) is a polyphenol extract derived from Ecklonia cava and dieckol (DK) is a major compound of SN. They have been known to exert various biological effects including anti-inflammatory and anti-oxidant effects $[3,4]$. A previous study demonstrated that the dieckol-rich extract of Ecklonia cava has an anti-diabetic effect against type 2 diabetes by improving glucose/lipid metabolism and the activity of antioxidant enzymes [5]. However, the mechanism of action underlying the antidiabetic effect of the major Ecklonia cava-derived compound has yet to be investigated.

Diabetes mellitus is one of the most common and serious metabolic diseases worldwide. Type 1 diabetes mellitus (insulin-dependent diabetes mellitus, IDDM) and type 2 diabetes mellitus (non-insulin-dependent diabetes mellitus, NIDDM) are the two main forms of diabetes. Of these, NIDDM accounts for $>90 \%$ of diabetes cases [6]. NIDDM is caused by obesity, genetic problems, and poor lifestyle habits such as a lack of exercise $[7,8,9]$. It is characterized by hyperglycemia and insulin resistance, caused by abnormal glucose uptake into cells. The development of NIDDM or chronic diabetes caused by hyperglycemia and insulin resistance can lead to diabetic complications including several vascular diseases, such as atherosclerosis, nephropathy, neuropathy, and peripheral artery disease [10]. Therefore, controlling hyperglycemia and insulin resistance could prevent or reduce diabetic complications and improve the quality of life of diabetic patients.

The objective of this study is to examine the effects of $\mathrm{SN}$ and its major compound, DK on diabetes and insulin sensitivity using a diabetic mice model and to determine the underlying mechanism of these effects. The current study showed the anti-diabetic effects of SN and its major compound DK in db/db mice by focusing on insulinrelated blood glucose levels and the insulin signaling pathway. 


\section{Materials and Methods}

\subsection{Materials}

Seapolynol and dieckol were obtained from Botamedi, Inc. (Jeju, South Korea). Antibodies against Akt, p-Akt, PI3K, and p-PI3K (p85 $\alpha)$ were purchased from Cell Signaling Technology (Danvers, MA, USA). Antibodies against IRS-1, p-IRS-1, GLUT4, and $\beta$-actin were obtained from Santa Cruz Biotechnology, Inc. (Santa Cruz, CA, USA). Dextrose anhydrate was obtained from JUNSEI (Chuo, Japan). Phosphate-buffered saline was purchased from iNtRON Biotechnology (Gyeonggi, Korea). Phosphatase inhibitor cocktails I and II and all other chemicals and reagents were purchased from Sigma (St. Louis, MO, USA).

\subsection{Animal Care and Experimental Protocol}

Male C57BL/KsJ-db/db mice were purchased from Joong-Ang Experimental Animal Co. (Seoul, Korea) and were housed at $21 \pm 2.0^{\circ} \mathrm{C}$ with $50 \pm 5 \%$ relative humidity under 12-h light/dark cycles. All mice were fed a commercial chow diet and tap water ad libitum for 1 week prior to being divided into the following experimental groups ( $\mathrm{n}=8$ /group): $\mathrm{db} / \mathrm{db}$, Rosi (rosiglitazone), $\mathrm{SN}$ (SNLD, low dose administration of SN, $60 \mathrm{mg} / \mathrm{kg}$ BW/day and SNHD, high dose administration of SN, 150 $\mathrm{mg} / \mathrm{kg}$ BW/day) and DK (DKLD, low dose administration of DK, $30 \mathrm{mg} / \mathrm{kg} \mathrm{BW/day} \mathrm{and} \mathrm{DKHD,} \mathrm{high} \mathrm{dose}$ administration of DK, $120 \mathrm{mg} / \mathrm{kg}$ BW/day). The normal diet was a purified form of Purina Laboratory Rodent Diet 38057 (Dyets Inc., Bethlehem, PA, USA). At the end of the experimental period (6 weeks), the animals were anesthetized using ethyl ether following a 12-h fast. To analyze plasma lipid levels, blood was drawn into EDTAcoated tubes via cardiac puncture. The plassma was obtained by centrifuging the blood sample at $2000 \mathrm{~g}$ for $15 \mathrm{~min}$ at $20^{\circ} \mathrm{C}$. The epididymis was removed, rinsed with PBS, and weighed. The plasma and visceral fat pad samples were collected and stored at $-80^{\circ} \mathrm{C}$ until analysis. All experimental mice were housed in a specific pathogenfree facility at the Korean Food Research Institute, Seongnam, Korea. The project was approved by the
Institutional Animal Care and Use Committee of CHA University (IACUC140054).

\subsection{Biochemical Analysis and Hepatic Lipid Isolation}

The plasma concentrations of total cholesterol, highdensity lipoprotein (HDL) cholesterol, and triglycerides were determined enzymatically using commercial kits (Asan Pharmaceutical, Gyeonggi, Korea). Fasting blood glucose concentrations were determined from the tail vein using a glucometer (Abbott Laboratories, MA, USA) every week after a 12-h fast. The levels of plasma insulin, adiponectin, and C-peptide were also measured using commercially available kits (Shibayagi Inc., Japan). The hepatic lipids were isolated using the method described by Folch et al. [11] with some modifications.

\subsection{Western Blotting}

Differentiated or undifferentiated cells were harvested in lysis buffer containing protease inhibitors and phosphatase inhibitor cocktails I and II. Protein extracts (30 or $50 \mu \mathrm{g}$ ) were separated using SDS-PAGE and transferred to PVDF (polyvinylidene fluoride) membranes. The membranes were blocked using 5\% non-fat dried milk and immunoblotted with specific primary antibodies overnight. The respective horseradish peroxidaseconjugated secondary antibodies (1:1000) were applied for $1 \mathrm{~h}$, and the bands were visualized using enhanced chemiluminescence and LAS imaging software (Fuji, New York, NY, USA).

\subsection{Statistical Analysis}

All data are expressed as the means \pm standard error of the mean. SAS 9.0 software was used for statistical analysis. One-way analysis of variance was used for comparisons among groups. Significant differences between the means were assessed using Duncan's test, and $\mathrm{P}$ values $<0.05$ were considered to be statistically significant.

\section{Results and Discussion}

Table 1. Bodyweight, food intake, water intake, bodyweight gain, and food efficiency ratio in $\mathrm{db} / \mathrm{db}$ mice

\begin{tabular}{|c|c|c|c|c|c|c|}
\hline Groups & $\mathrm{db} / \mathrm{db}$ & $\mathrm{db} / \mathrm{db}$ & & & & \\
\hline Variables & & Rosi & SNLD & SNHD & DKLD & DKHD \\
\hline Initial body weight (g) & $38.13 \pm 2.34$ & $38.25 \pm 2.15$ & $39.66 \pm 1.51$ & $38.79 \pm 2.91$ & $38.23 \pm 3.38$ & $38.59 \pm 2.35$ \\
\hline Final body weigh (g) & $55.38 \pm 2.1 b$ & $58.2 \pm 2.41 \mathrm{a}$ & $54.04 \pm 1.99 b$ & $49.06 \pm 3.91 \mathrm{c}$ & $51.8 \pm 2.64 b$ & $51.8 \pm 3.4 \mathrm{~b}$ \\
\hline Food intake (g/day) & $7.35 \pm 0.61$ & $6.78 \pm 0.63$ & $7.04 \pm 0.66$ & $7.78 \pm 0.37$ & $7.4 \pm 0.38$ & $7.4 \pm 0.63$ \\
\hline Water intake (mL/day) & $4.64 \pm 0.38 \mathrm{a}$ & $1.5 \pm 0.24 \mathrm{c}$ & $3.58 \pm 0.36 b$ & $3.7 \pm 0.37 b$ & $4.3 \pm 0.53 a$ & $3.71 \pm 0.48 b$ \\
\hline Body weight gain (g/week) & $3.45 \pm 0.39 b$ & $3.99 \pm 0.29 a$ & $2.88 \pm 0.24 \mathrm{c}$ & $2.18 \pm 0.55 d$ & $2.39 \pm 0.64 c$ & $2.63 \pm 0.69 c$ \\
\hline Food efficiency ratio & $0.046 \pm 0.002 b$ & $0.055 \pm 0.002 \mathrm{a}$ & $0.04 \pm 0.002 c$ & $0.027 \pm 0.009 \mathrm{~d}$ & $0.025 \pm 0.01 d$ & $0.031 \pm 0.002 \mathrm{~d}$ \\
\hline
\end{tabular}

\subsection{Food and Water Intake and Body Weight}

The initial bodyweight, food intake, and bodyweight gain of the Rosi, SN and DK groups were not significantly different from those of the $\mathrm{db} / \mathrm{db}$ control group (Table 1). However, the final body weights among the groups were different. Rosiglitazone, a negative control, slightly increased the final bodyweight $(58.71 \pm 1.71)$ compared with the $\mathrm{db} / \mathrm{db}$ control group. Conversely, SNHD (49.24 \pm 3.52$)$ and DKHD $(50.32 \pm 1.71)$ treatments significantly decreased the final bodyweight compared with the $\mathrm{db} / \mathrm{db}$ control group (55.38 \pm 1.82$)$. Water intake was significantly decreased in the Rosi, SNHD, and DKHD groups compared with the $\mathrm{db} / \mathrm{db}$ control group. In addition, the food efficiency ratio (FER, total weight gain/total food intake) was significantly decreased in the SN and DK groups supplementation; SNHD reduced the FER by $49 \%$, whereas DKLD and DKHD decreased the FER by $54 \%$ and $67 \%$, respectively (Table 1 ). The decrease in water intake in the Rosi and SN/DK groups was also observed in Lee's study [5]. However, the 
SN/DK-mediated decrease in the final body weight in $\mathrm{db} / \mathrm{db}$ mice was shown to be different from that of the study performed by Lee et al, which did not show a significant difference with the supplementation of the dieckol-rich extract of Ecklonia cava.

\subsection{Blood Glucose Levels}

SNLD (14.9 mmol/L), SNHD (13.9 mmol/L), and DKHD (14 mmol/L) treatments significantly reduced fasting blood glucose levels compared with the $\mathrm{db} / \mathrm{db}$ control group (20.3 mmol/L) after 6 weeks (Figure 1a). In particular, the Rosi $(4.96 \mathrm{mmol} / \mathrm{L})$ group had much lower fasting blood glucose level than the control group (Figure 1a). This result was supported by the difference in the variation of the fasting glucose levels, which represented the difference between the fasting blood glucose levels of the 5-week-old mice before treatment and the 11-week-old mice after treatment (Figure 1b). We also observed that SN and DK significantly suppressed the blood glucose levels in the oral glucose and maltose tolerance tests.

\subsection{Plasma Insulin, C-peptide, and Adiponectin Levels and HOMA-IR}

In addition, we measured plasma insulin (Figure 1c) and C-peptide levels (Figure 1d). Rosi markedly reduced plasma insulin levels compared with the $\mathrm{db} / \mathrm{db}$ control group. SN and DK treatments induced a dose-dependent reduction of plasma insulin levels (Figure 1c). High-doses of SN and DK decreased plasma insulin levels by approximately $28 \%$ compared with the $\mathrm{db} / \mathrm{db}$ control group. Similarly, SN and DK treatments reduced plasma C-peptide levels in a dose-dependent manner (Figure 1d). SNHD and DKHD treatments lowered plasma C-peptide levels by $44 \%$ compared with the control group. This result indicates that SN and DK reduced insulin secretion, thus responding to plasma glucose levels.

On the other hand, plasma adiponectin levels were significantly increased by SN and DK in a dose-dependent manner (Figure 1e). In particular, SNHD and DKHD treatments increased plasma adiponectin levels by more than two-fold compared with the diabetic mice group, showing a higher level than the Rosi group (Figure 1e) The HOMA-IR, which refers to insulin resistance, was significantly decreased in the SN and DK groups, an effect that was dose dependent. These results indicate that SN and DK control increases in the plasma glucose levels by regulating insulin sensitivity. C-peptide is a indicator of insulin secretion and its half-life is known to be longer than that of plasma insulin [12]. Therefore, C-peptide levels provide more specific and accurate data regarding insulin secreted in mouse blood. The SN/DK-mediated reduction in the levels of $\mathrm{C}$-peptide was correlated with the decrease in plasma insulin levels (Figure 1c).

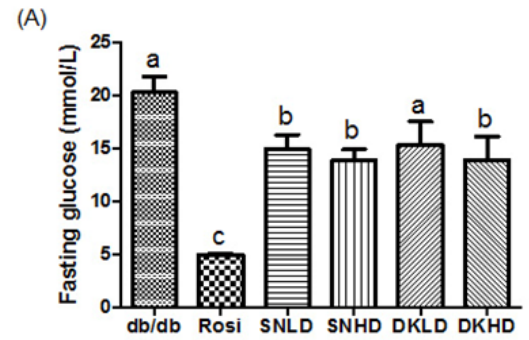

(C)

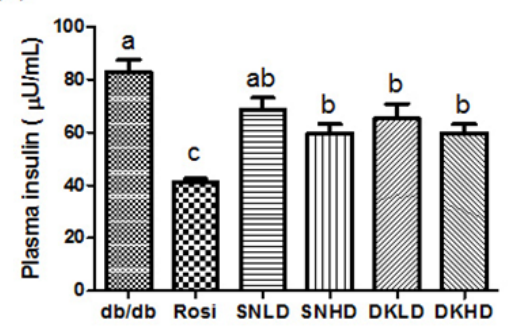

(E)

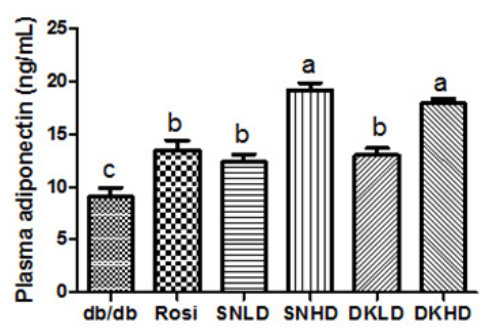

(B)

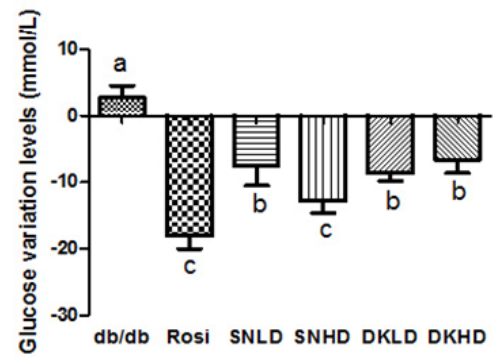

(D)

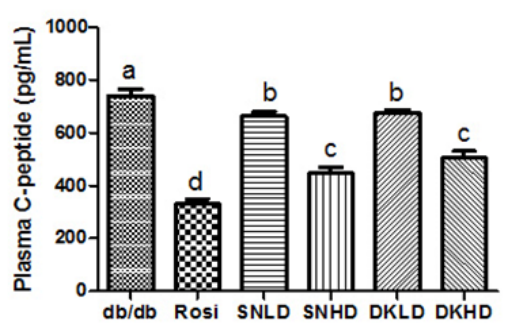

(F)

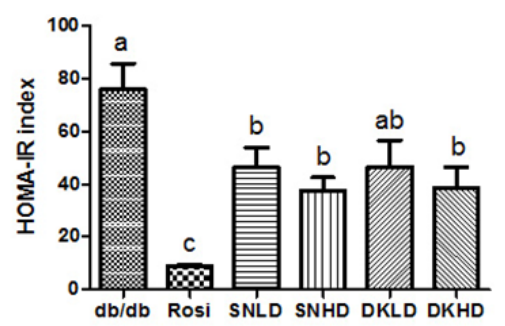

Figure 1. Effects of SN and DK on fasting glucoselevels, variations in fasting glucose levels, plasma insulin levels, C-peptide levels, adiponectin levels, and the HOMA-IR index in $\mathrm{db} / \mathrm{db}$ mice

Serum fasting glucose levels were measured after 6 weeks of treatment (A). The variations in fasting glucose levels were calculated by subtracting the blood glucose levels of non-treated mice from those of the 6-week treatment mice (B). Plasma insulin (C), C-peptide (D), and adiponectin levels (E) were measured at the end of the 6-week treatment. The HOMA-IR index (F) was calculated as follows:

fasting glucose $(\mathrm{mmol} / \mathrm{L}) \times$ fasting insulin $(\mathrm{IU} / \mathrm{mL}) / 22.5$

Data are presented as the means \pm SEM from three replicates. Means not designated by a common superscript are significantly different $(p<0.05)$. 
Adiponectin is a $30-\mathrm{kDa}$ collagen-like plasma protein that functions as an insulin-sensitizer in adipose tissue [13]. The SN/DK-induced increase in adiponectin agreed with the decrease in fasting glucose and insulin levels, suggesting that SN/DK improves insulin sensitivity in the diabetic condition. This beneficial effect of SN/DK on the adiponectin level was supported by the decrease in the HOMA-IR level. Therefore, our data indicate that SN/DK improves insulin sensitivity to regulate plasma glucose levels.

\subsection{Plasma and Hepatic Lipid Profiles}

The effects of SN and DK on plasma lipid profiles were determined by measuring triacylglycerol, total cholesterol, HDL-cholesterol, and LDL-cholesterol levels. The levels of plasma triacylglycerol were significantly decreased in the Rosi, SNHD, and DKHD treatment groups compared with the diabetic control group (Table 2). In addition, the Rosi, SN, and DK treatments lowered the total and LDL cholesterol levels significantly compared with the $\mathrm{db} / \mathrm{db}$ control group. SN/DK-induced decrease in the triglyceride and total cholesterol levels was also observed in liver. However, the level of HDL cholesterol was shown to be slightly increased in the groups treated with high-doses of SN and DK (Table 2). These results were also observed in Lee's study, which used a dieckol-rich extract [5].

Table 2. Plasma and hepatic lipid profiles

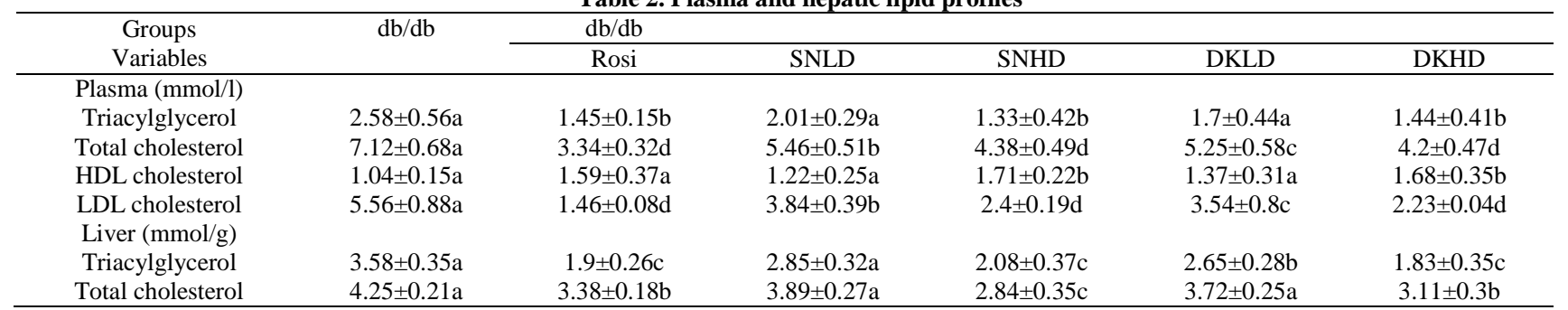

In many studies, body weight and lipid parameters such as, total cholesterol, LDL cholesterol, and triacylglycerol was shown to be increased in diabetes [14]. In particular, Rosiglitazone, an effective agent for the treatment of hyperglycemia [15], has included lipid accumulation and body weight gain as a side effect [16]. Thus, SN/DK, which have shown desirable effects on the lipid profile and body weight gain in diabetic condition, have been considered to have a potential as desirable anti-diabetic agents.

\subsection{Effects of SN and DK on Insulin Signaling Pathways}

We examined the effects of SN and DK on the expression of various factors related to insulin signaling in the epididymal fat of $\mathrm{db} / \mathrm{db}$ mice. Treatment with Rosi, $\mathrm{SN}$, and DK induced the activation of insulin signaling pathways by increasing the phosphorylation of IRS-1,
PI3-kinase, and Akt. The SN/DK-induced activation of insulin signaling was clearly observed in the high-dose groups (Figure 2a and b). In addition, GLUT4, a transporter of glucose, was upregulated in the SN/DK and Rosi groups, suggesting that SN/DK control plasma glucose levels by regulating the expression of GLUT4. The correlation of IRS-1/PI3K/Akt signaling cascades and glucose uptake mechanisms is well known $[17,18]$.

The insulin-stimulated phosphorylation of IRS-1 leads to the gradual activation of PI3-kinase and Akt, followed by a signaling cascade that results in glucose uptake, fatty acid synthesis, and glycogen synthesis $[7,19]$. Therefore, the administration of SN and DK was shown to stimulate glucose uptake via GLUT4, and the upregulation of GLUT4 was shown to be due to the activation of the insulin signaling pathway by SN/DK. This suggests that $\mathrm{SN}$ and DK reduced blood glucose levels by regulating insulin signaling pathways in $\mathrm{db} / \mathrm{db}$ mice.
(A)

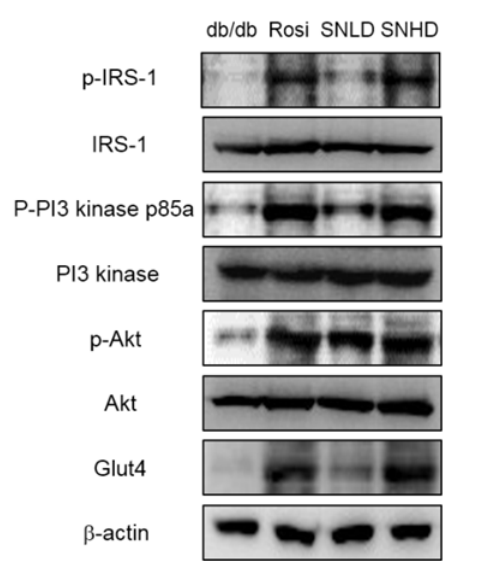

(B)

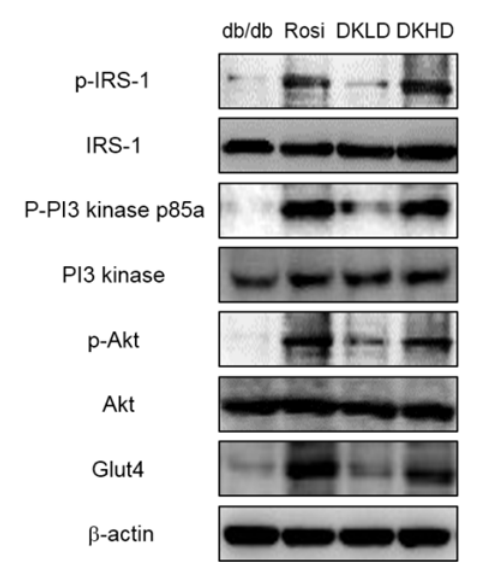

Figure 2. SN regulates the IRS signaling pathways and GLUT4 in $\mathrm{db} / \mathrm{db}$ mice

Proteins were collected from the epididymal fat of db/db mice after 6 weeks of treatment. SN (A) and DK (B) increased the phosphorylation of IRS-1 (p-IRS-1), p-PI3K, and p-Akt as well as GLUT4 expression. Protein levels were measured by Western blotting. All data are representative of three replicates. Means not designated by a common superscript are significantly different $(p<0.05)$. 


\section{Conclusion}

In conclusion, SN and DK control blood glucose and lipid levels by improving insulin sensitivity in $\mathrm{db} / \mathrm{db}$ mice. The SN/DK-mediated increase in insulin sensitivity was considered to be due to the activation of IRS-1/PI3K/Akt signaling. Thus, SN and DK may be useful in the prevention of diabetic complications as therapeutic agents for type 2 diabetes mellitus.

\section{Conflict of Interest}

The authors declare that they have no conflict of interest.

\section{Acknowledgments}

This research was a part of the project entitled "Studies on adipogenic signaling pathways regulated by phytochemicals from edible agricultural and marine products." funded by the Ministry of Education and Science Technology, Korea, to Boo-Yong Lee and "2013 RIA1A2064024” funded by National Research Foundation of Korea to Boo-Yong Lee.

\section{References}

[1] Li Y, Qian ZJ, Ryu B, Lee SH, Kim MM, Kim SK. Chemical components and its antioxidant properties in vitro: an edible marine brown alga, Ecklonia cava. Bioorganic \& medicinal chemistry 2009; 17:1963-73.

[2] Kim JH, Lee NS, Jeong YG, Lee JH, Kim EJ, Han SY. Protective efficacy of an Ecklonia cava extract used to treat transient focal ischemia of the rat brain. Anatomy \& cell biology 2012; 45:10313.

[3] Athukorala Y, Kim KN, Jeon YJ. Antiproliferative and antioxidant properties of an enzymatic hydrolysate from brown alga, Ecklonia cava. Food and chemical toxicology: an international journal published for the British Industrial Biological Research Association 2006; 44:1065-74.

[4] Jung WK, Heo SJ, Jeon YJ, Lee CM, Park YM, Byun HG, et al. Inhibitory effects and molecular mechanism of dieckol isolated from marine brown alga on COX-2 and iNOS in microglial cells. Journal of agricultural and food chemistry 2009; 57:4439-46.

[5] Lee SH, Min KH, Han JS, Lee DH, Park DB, Jung WK, et al. Effects of brown alga, Ecklonia cava on glucose and lipid metabolism in C57BL/KsJ-db/db mice, a model of type 2 diabetes mellitus. Food and chemical toxicology: an international journal published for the British Industrial Biological Research Association 2012; 50:575-82.

[6] Zimmet P, Alberti KG, Shaw J. Global and societal implications of the diabetes epidemic. Nature 2001; 414:782-7.

[7] Kahn SE, Hull RL, Utzschneider KM. Mechanisms linking obesity to insulin resistance and type 2 diabetes. Nature 2006; 444:840-6.

[8] Sladek R, Rocheleau G, Rung J, Dina C, Shen L, Serre D, et al. A genome-wide association study identifies novel risk loci for type 2 diabetes. Nature 2007; 445:881-5.

[9] Tuomilehto J, Lindstrom J, Eriksson JG, Valle TT, Hamalainen H, Ilanne-Parikka $\mathrm{P}$, et al. Prevention of type 2 diabetes mellitus by changes in lifestyle among subjects with impaired glucose tolerance. The New England journal of medicine 2001; 344:134350.

[10] Beckman JA, Creager MA, Libby P. Diabetes and atherosclerosis: epidemiology, pathophysiology, and management. Jama 2002; 287:2570-81.

[11] Folch J, Lees M, Sloane Stanley GH. A simple method for the isolation and purification of total lipides from animal tissues. The Journal of biological chemistry 1957; 226:497-509.

[12] Knapczyk J. [Kinetics of sodium nitrite decomposition]. Acta poloniae pharmaceutica 1975; 32:683-9.

[13] Wang C, Mao X, Wang L, Liu M, Wetzel MD, Guan KL, et al. Adiponectin sensitizes insulin signaling by reducing p70 S6 kinase-mediated serine phosphorylation of IRS-1. The Journal of biological chemistry 2007; 282:7991-6.

[14] Hollander PA, Elbein SC, Hirsch IB, Kelley D, McGill J, Taylor T, et al. Role of orlistat in the treatment of obese patients with type 2 diabetes. A 1-year randomized double-blind study. Diabetes care 1998; 21:1288-94.

[15] Nathan DM, Buse JB, Davidson MB, Ferrannini E, Holman RR, Sherwin $\mathrm{R}$, et al. Medical management of hyperglycaemia in type 2 diabetes mellitus: a consensus algorithm for the initiation and adjustment of therapy: a consensus statement from the American Diabetes Association and the European Association for the Study of Diabetes. Diabetologia 2009; 52:17-30.

[16] Young PW, Buckle DR, Cantello BC, Chapman H, Clapham JC, Coyle PJ, et al. Identification of high-affinity binding sites for the insulin sensitizer rosiglitazone (BRL-49653) in rodent and human adipocytes using a radioiodinated ligand for peroxisomal proliferator-activated receptor gamma. The Journal of pharmacology and experimental therapeutics 1998; 284:751-9.

[17] Esposito DL, Li Y, Cama A, Quon MJ. Tyr (612) and Tyr (632) in human insulin receptor substrate-1 are important for full activation of insulin-stimulated phosphatidylinositol 3-kinase activity and translocation of GLUT4 in adipose cells. Endocrinology 2001; 142:2833-40.

[18] Kim YB, Nikoulina SE, Ciaraldi TP, Henry RR, Kahn BB. Normal insulin-dependent activation of Akt/protein kinase B, with diminished activation of phosphoinositide 3-kinase, in muscle in type 2 diabetes. The Journal of clinical investigation 1999; 104:733-41.

[19] Saltiel AR, Kahn CR. Insulin signalling and the regulation of glucose and lipid metabolism. Nature 2001; 414:799-806. 